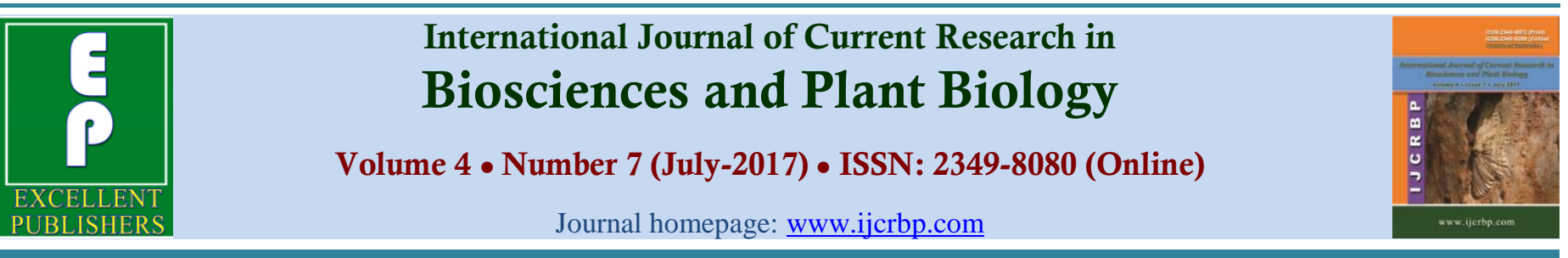

\title{
Brassinosteroids - An Essential Steroidal Regulator: Its Structure, Synthesis and Signaling in Plant Growth and Development- A Review
}

\author{
Heena Khatoon ${ }^{1}$, Ananya Singh ${ }^{2}$, Farhan Ahmad ${ }^{1}$ and Aisha Kamal ${ }^{1 *}$ \\ ${ }^{I}$ Department of Bioengineering, Integral University, Dasuli, Kursi Road, Lucknow-226 026, Uttar Pradesh, India \\ ${ }^{2}$ Department of Biosciences, Integral University, Dasuli, Kursi Road, Lucknow-226 026, Uttar Pradesh, India
}

*Corresponding author.

\begin{abstract}
Brassinosteroids (BRs) are growth-initiating steroidal compound found in pollen, seeds, and young vegetative tissues throughout the plant kingdom. Brassinosteroids (BRs) collectively refers to naturally-occurring $5 \alpha$ cholestane steroids that elicit growth stimulation in nano- or micromolar concentrations. BRs that are biosynthesized using sterols as precursors are structurally similar to the cholesterol-derived, human steroid hormones and insect molting hormones. BRs have been known for decades to be effective in plant growth promotion. However, definitive evidence for their roles in growth and development remained unclear until the recent characterization of BR dwarf mutants isolated from Arabidopsis and other plants. This review inspects essential role of BRs in diverse developmental programs, including cell expansion, vascular differentiation, etiolation, and reproductive development. Current improvements regarding BR-regulated gene expression and signal transduction are also discussed.
\end{abstract}

\section{Article Info}

Accepted: 16 June 2017

Available Online: 06 July 2017

\section{Keywords}

Brassinosteroids

Physiological effect

Signaling pathway

Steroidal compounds

\section{Introduction}

Brassinosteroids (BRs) polyhydroxy steroids play an essential role in plant development. The origin of BRs research started with discovery of lipophilic compound, rape pollen extracts of Brassica napus (Mitchel et al., 2009). BRs have been broadly accepted as the sixth class of essential plant hormones. Brassinolide (BL) is the most bioactive form of growth promoting plant steroids termed as Brassinosteroids (BRs). At present 70 such analogues (65 unconjugated and 5 conjugated) have been identified from 60 plant species including 51 angiosperms (12 monocotyledons and 38 dicotyledons), 1-pteridophyte (Equisetum arvense), 1 bryophyte
(Marchantia polymorpha) and 1 chlorophyte and rest gymnosperms (Hayat and Ahmad, 2011). Their multiple effects they can affect general plant and many developmental process such as seed germination, rhizogenesis, flowering senescense, abscission and maturation, leaf bending and epinasty. Circulation of BRs is not even throughout the plant body; however, young growing tissues have relatively a large share than the mature tissues (Yokota and Takahashi, 1986). Now, these are known to be present in all plant parts, including roots (Bajguz and Tretyn, 2003).

Brassinosteroids (BRs) a class of steroidal phytohormones occur in all plant parts, including roots 
that brings out a wide range of physiological responses in plants; including stem elongation, pollen tube growth; leaf bending; root growth inhibition; induction of ethylene biosynthesis; activation of proton pump;xylem differentiation and also speed up the rate of photosynthesis(Hayat et al, 2010). Brassinosteroids are involved in the regulation of various developmental and physiological processes. They promote cell division, cell wall regeneration, cell expansion and elongation (Clouse and Sasse, 1998) and interplay with auxins (Nemhauser et al., 2004). BRs are also necessary for the promotion of vascular differentiation (Caño-Delgado et al., 2004), pollen tube formation and elongation (Hewitt et al., 1985). Furthermore, BRs delayed senescence in BRs deficient mutants, while accelerated senescence in dying tissue that signifies the biological relevance of BRs action (Clouse and Sasse, 1998). BRs counteract abiotic stresses in plants (Sharma and Bhardwaj, 2007) while it inhibit pathogen-associated molecular pattern (PAMP)triggered immune signaling (Albrecht et al., 2012).

\section{Chemical structure and occurrence}

BRs were found in all plant organs such as pollen, anthers, seeds, leaves, stems, roots, flowers, and grain. Other tissues where the presence of BR is detected are insect and crown galls, for example the galls of Castanea crenata, Distylium racemosum or Catharanthus roseus (Bajguz and Tretyn, 2003). These plants have higher levels of BRs than the normal tissues. Brassinosteroids are classified in C27, C28 and C29 structure.

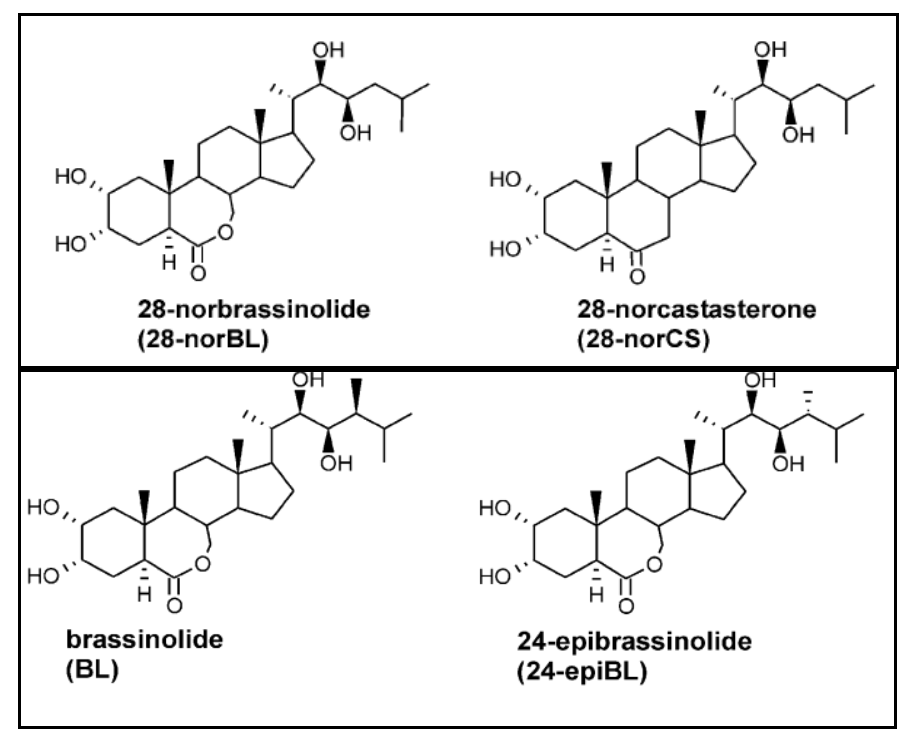

Fig. 1: Chemical structures of C28 brassinosteroids.

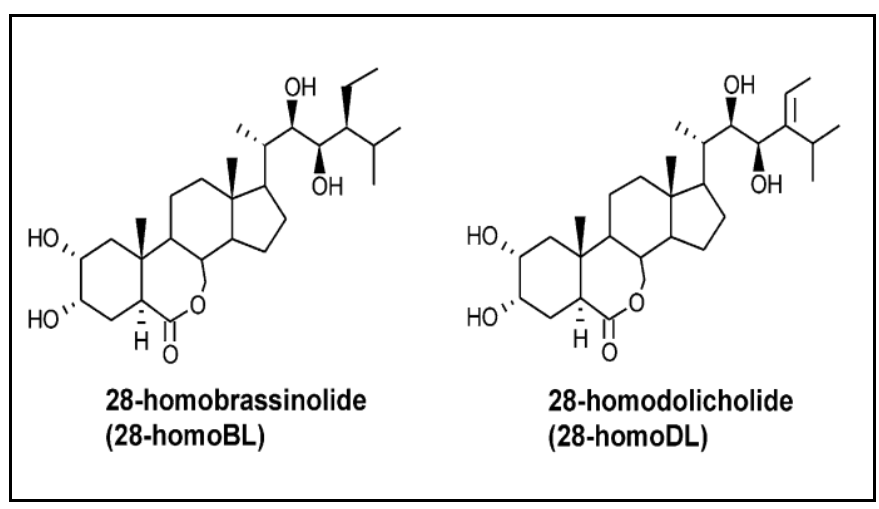

Fig. 2: Chemical structures of $\mathrm{C}_{29}$ brassinosteroids.

\section{Biosynthesis}

Brassinosteroids are steroidal hormones derived from cholesterol. Among plant sterols, campesterol or its analogues areassumed to be the biosynthetic precursors of brassinolide based on the identity of the side-chain skeleton (Suzuki et al., 1995). BR biosynthetic pathway was largely established utilizinggas chromatographymass spectrometry (GC-MS) to identifymetabolic products and their reaction sequence after feeding Catharanthus roseus suspension cultures or Arabidopsis seedlings with various isotope-labeled intermediates (Zhao and Li, 2012).

The brassinosteroid-deficient mutants and the cloned stronger activity in promoting hypocotyl elongation of brassinosteroid-biosynthetic genes provide an important dark-grown seedlings tool for investigating not only the physiological functions, but also the biosynthetic pathways of Brassinosteroids ( $\mathrm{Li}$ and Chory, 1999). Detailed study of the biosynthesis of BL, a C28 BR, revealed that two parallel pathways are operative, the early and late C6 oxidation pathways (Fukaki et al., 1998).

In the sequential biosynthetic pathway from campesterol to brassinolide, C6 oxidation occurs before theoxidation of the side chain and A ring. Cathasterone is hydroxylated at the $\mathrm{C} 23$ position to yield teasterone, a brassinosteroid frequently found in plants $\sim 3$; teasterone is then converted to typhasterol. Conversion of teasterone to castasterone is known to occur in intact plants of $C$. roseus, tobacco and rice. Conversion of castasterone to brassinolide is also observed in the seedlings of $C$. roseus TM. It therefore appears that the early C6 oxidation pathway operates in intact tissues. Below figure gives a detailed description of early and late C6 oxidation pathways. 


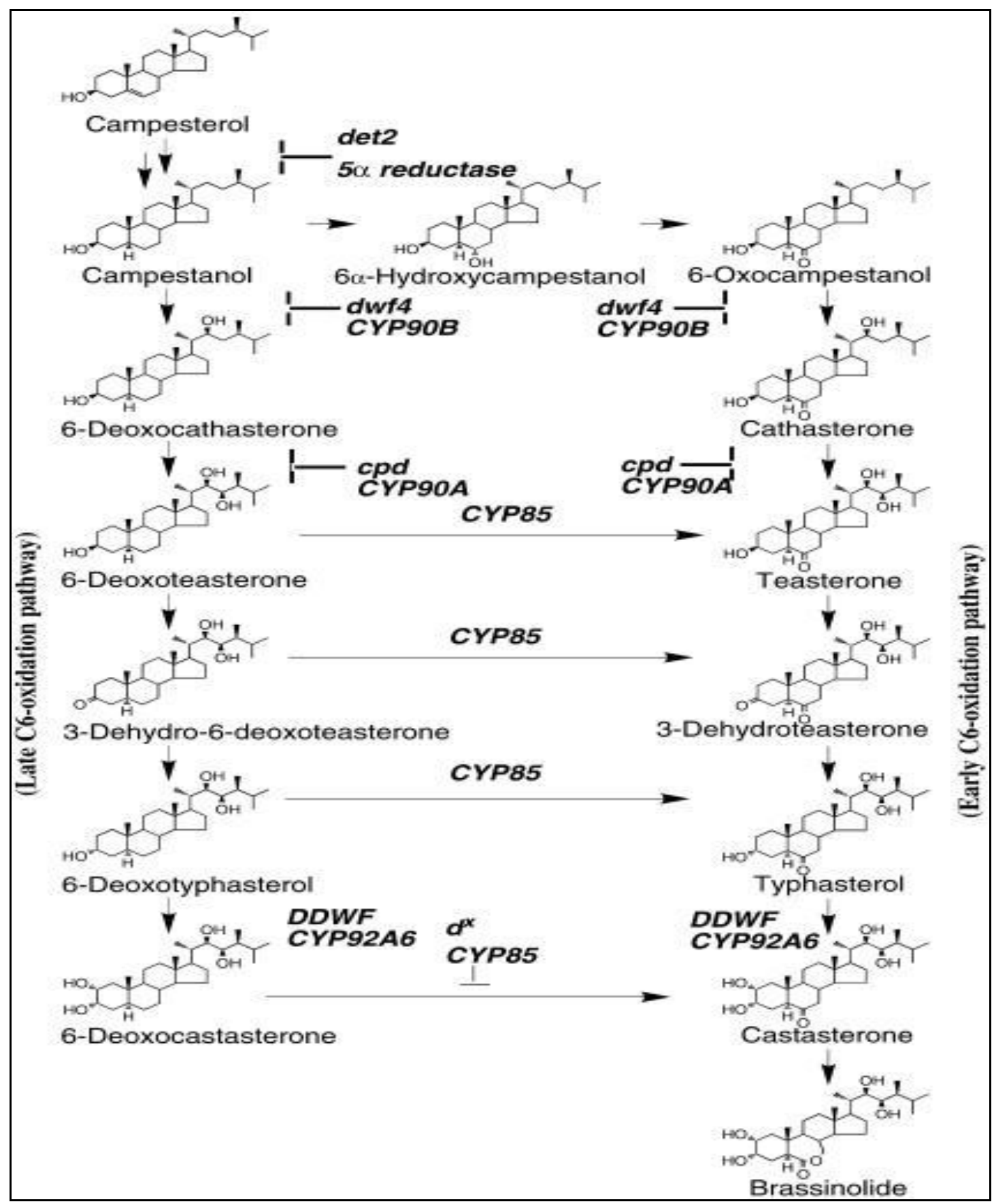

Fig. 3: Simplified brassinosteroid biosynthetic pathway with enzymes involved in each of the reactions.

\section{Signalling}

The Brassinosteroids (BR) class of steroid hormones regulates plant development and physiology. Recent studies have totally connected the BR signal transduction chain and have established thousands of BR target genes, linking BR signaling to numerous cellular processes (Zhu and Wang, 2013). BR signaling cascade involves the perception of the BR signal. On
BR activation, BRI1 phosphorylates members of two groups of plasma membrane-anchored cytoplasmic kinases represented by brassinosteroid signallingKinase 1 and constitutive differential growth1 (CDG1) (Kim et al., 2011; Tang et al., 2008). It has been perceived by BRI1, a leucine-rich repeat (LRR) which functions with its coreceptor BAK1/SERK3 in BR signaling, they are arranged in super helix shape. Overexpression of BR biosynthetic enzymes and the 
BRI1 receptor increases cell elongation and plant growth, various intensive research work have elucidated the roles of BR signaling elements regarding the overall plant growth. The genetic studies have revealed identification of $\mathrm{BR}$ receptor and downstream signaling component. BRI1 is an LRR receptor-like kinase (LRR-RLK) located on the cell surface. It has an extracellular domain containing 25 LRRs, a transmembrane domain, and acytoplasmic serine/threonine kinase domain. BRI1 immunoprecipitates with BR binding activity and BR induce auto-phosphorylation ofBRI1 in vivo. BAK1 is potentially another component of the $\mathrm{BR}$ receptor complex. BAK1 interacts with BRI1 in vitro and in vivo environment, and phosphorylation takes place in vitro condition. The result of gain and loss of function experiments support a positive role for BAK1 in BR signaling.

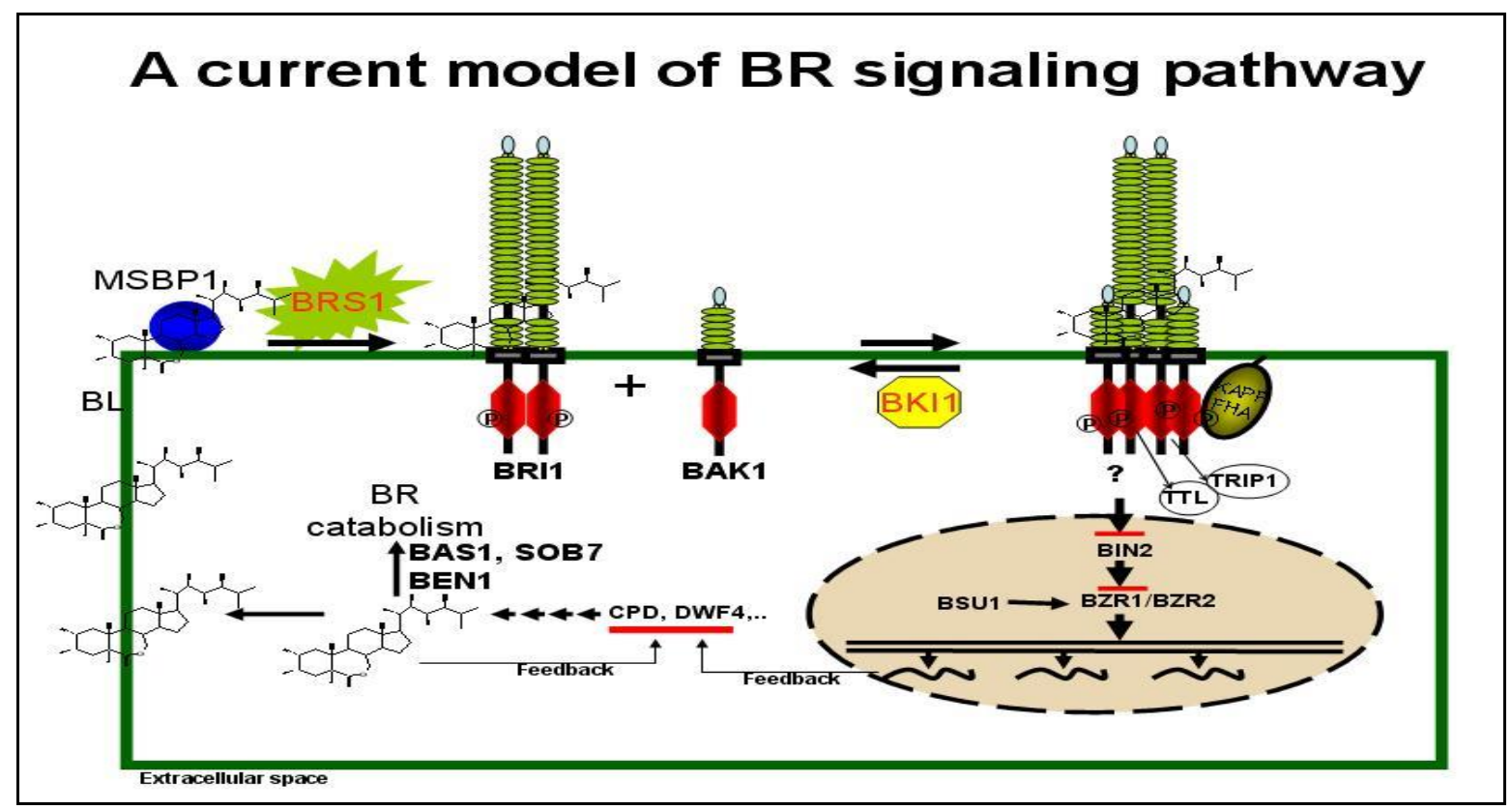

Fig. 4: BR Signalling model.

These models show the effect of ABA on BR signaling pathway output. For BR signaling pathway BR binding to BRI1 which activate BRI1 complex by releasing plasma membrane association of BKI1.Gain of function mutation of $\mathrm{ABI} 1$ and $\mathrm{ABI} 2$ in the $\mathrm{ABA}$ early signaling can restrain the effect of $\mathrm{ABA}$ on $\mathrm{BR}$ signaling. In the absence of $\mathrm{BR}$, or when BR levels are lowered then active BIN2 shows phosphorylation of two homologous transcription factors, BZR1 and BZR2 (brassinazole resistant1) [also named BRI1-EMS-SUPPRESSOR1 (BES1)] (He et al., 2002) to abate their DNA-binding activities and cause their cytoplasmic retention by . As the BR levels are increased, B2N2 is inactivated by BSU1 and degraded by the proteasome (Peng et al., 2008).

BZR1 and BZR2 are dephosphorylated by PROTEIN PHOSPHATASE 2A (PP2A) (Tang et al., 2011). A smaller set of targets, which overlaps significantly with the BZR1 targets, has been recognized for BZR2 (Gudesblat and Russinova, 2011). These BZR1 or BZR2 target genes have revealed numerous molecular links to specific cellular, metabolic and developmental processes. Particularly, cell wall modification and cellular transport are major cellular functions which are targeted by BR, stabilized with its effects on cell elongation and growth (BKI1 function in brassinosteroid signaling was explored in various ways. First, we had made a transgenic plant which has harboured a bglucuronidase (GUS) reporter gene expressed by the promoter of BKI1 for observation of the expression pattern of BKI1 during development. BKI1 was expressed in leaves, petioles, shoot apices, hypocotyls, and flowers, indicating that BKI1 and BRI1 are coexpressed in a number of tissues. To traverse the function of BKI1 in BRI1 signaling, RNA interference (RNAi) lines was created to restrain BKI1 RNA levels (Sun et al., 2010). Real-time polymerase chain reaction (RT-PCR) analysis indicates that the transcript level of lines as compared with a control line. The RNAi lines had longer hypocotyls than the control line which was grown in short days. 


\section{Physiological roles}

\section{Effect of brassinosteroids on seed germination}

BRs involvement in the regulation of seed germination is well-accepted. BRs treatment to the seeds of Lepidium sativus (Jones-Held et al., 1996) and Eucalyptus camaldulensis (Sasse, 2003) improved percent germination. Similarly BRs promoted seed germination in case of Pisum sativum (Nomura et al., 2007) and Arabidopsis (Steber and McCourt, 2001). In A. thaliana, BRs promoted the germination of pre-chilled (i.e. non-dormant) seeds of BRs-deficient biosynthesis mutant det2-1 and the BRs insensitive response mutant bri1-1 imbibed in the light (Steber and McCourt, 2001). Interestingly, a new allele sly 1 was identified in a screen for BR dependent germination and also proposed an interaction between BRs and GA signaling in seeds (Bai et al., 2012). Heavy metal pollution has developed ecological crisis. Industrial pollution by heavy metals such as $\mathrm{Cr}, \mathrm{Mn}, \mathrm{Co}, \mathrm{Ni}, \mathrm{Cu}, \mathrm{Zn}$ and $\mathrm{Hg}$ is harmful not only to human beings, but also to all living organisms. Brassinosteroids have been recommended to decrease the absorption and accumulation of heavy metals and radioactive elements in plants (Bajguz, 2000; Kaur and Bhardwaj, 2004). It was observed that the low concentrations of brassinosteroids applied at a certain stage of development reduced the metal absorption in barley, tomatoes and sugar beet significantly (Volynets et al., 1997).

\section{Cell division}

Restoration of leaf size after subjecting of brassinolide to the mutants det 2 and dwf 1 of Arabidopsis could not be accounted for solely by expansion, supporting a role for BR in cell division (Nakaya et al., 2002). Significant interactions with auxin at very low concentration of brassinolide were also observed (Bajguz and Tretyn, 2003) so this single -cell system has potential for exploring hormones. Understanding the regulatory mechanisms of brassinosteroid (BR) biosynthesis in particular plant developmental processes, we first investigated the accumulation profiles of BRs and sterols in xylem differentiation in a Zinnia culture. The amounts of substances in the late $\mathrm{C} 28$ sterol biosynthetic pathway to campesterol (CR), such as episterol and 24methylenecholesterol, including those in the BR-specific biosynthetic pathways from CR to brassinolide (BL), were promoted in close association with tracheary element differentiation. Xylem differentiation is example of BR dependent development (Fukuda, 2004). BRI1 is expressed in growing cells, other three members BRL1, BRL2 and BRL3 of the BRI1 family show preferential vascular cell expression (Clay and Nelson, 2002). The in-vitro process of xylem cell differentiation in Zinnia cell culture is divided into three stages. Stage 1 corresponds to the functional dedifferentiation process from mesophyll cell to dedifferentiated cells. In stage 2 dedifferentiated cells may change into tracheary element precursor cells. Stage 3 it involves treachery element specific events such as secondary wall formation and cell death.BR biosynthesis inhibitors suppresses the gene expression during stage (Yamamoto et al., 1997). Increase in seed germination was observed to be maximum in28-HBLas compared to untreated control seeds. 24-EBL was found to be the best concentration for seed germination.

\section{Cell expansion}

Cell expansion, plays a critical role in the growth and development of plant organs. However, the genes controlling cell expansion during organogenesis are largely unknown. In contrast to cell division, cell expansion seems to affect organs growth in more complicated ways. Brassinosteroids is an important plant hormone required for normal cell (Clouse and Sasse, 1998). Here, we demonstrate that a novel Arabidopsis gene, ARGOS-LIKE (ARL), having rare sequence homology to the ARGOS gene is involved in this process. Reduced expression or overexpression of ARL in Arabidopsis results in smaller or larger cotyledons and leaves as well as other lateral organs, respectively. Cell elongation and expansion in plants are regulated by plant hormones, such as auxin, gibberellic acid (GA), ethylene and brassinosteroids (BR). These signals may regulate cell expansion and differentiation through their specific pathways, thus influence in organ growth or size (Mizukami, 2001). For instance, ABP1, are essential for auxin-regulated cell expansion (Jones-Held et al., 1996). Coordinated plant growth is modulated through networked actions of plant growth regulators. The possibility that BRs may control aquaporin activities was explored using dwarf biosynthetic and sensitivity mutants cpd and bril of Arabidopsis. Plant growth is largely accomplished by cell expansion which depends on the de-novo biosynthesis and modification of cell wall components. Crucial for anisotropic growth in expansion are polarization axialization and ongoing control of the orientation of microtubules and cellulose microfibrils. Critical requirement for cell expansion is 
water uptake and flow across membranes. Aquaporins helps in the intercellular and intracellular water transport in plants.

\section{Reactive oxygen species}

Reactive Oxygen Species (ROS) are continuously assembled during seed development, from embryogenesis to germination, but also during seed storage. ROS play a dual role in seed physiology behaving, as actors of cellular signaling pathways on one hand and, on the other as, toxic products that accumulate under stress conditions (Bailly et al., 2008). ROS, they do not act as harmful compounds and instead their fine-tuning plays a key role in the gene expression. It appears now as being beneficial for germination, and in particular it acts as a positive signal for seed dormancy release. Its effect might result from the interplay between ROS and hormone signaling pathways thus leading to changes in gene expression or in cellular redox status. It is also seen that changes in ROS homeostasis play a role in perception of environmental factors by seeds during their germination, and thus act as a signal controlling the completion of germination. However, the uncontrolled accumulation of ROS usually occurs during seed aging or seed desiccation thus leading to oxidative damage toward a wide range of biomolecules and ultimately to necrosis and cell death. The involvement of BRs in the regulation of reactive oxygen species (ROS) metabolism is evident as they can induce and regulate the expression of certain antioxidant genes and increase the activities of key antioxidant enzymes. Several reactive oxygen species (ROS) are continuously produced in plants as byproducts of aerobic metabolism. Based on the nature of the ROS species, some species are highly toxic and rapidly detoxified by various cellular enzymatic and nonenzymatic mechanisms.

\section{Induction of ehylene biosynthesis}

Brassinosteroids (BR) and indole 3-acetic acid (IAA) alone and in combination were evaluated for their effects on Ethylene and ACC synthase production in etiolated mung bean hypocotyls segments. BR induced ethylene can be inhibited by aminooxyacetic acid(AOA), fusicoccin (a fungal toxin)and the transport inhibitors 2,3,4- tri-iodobenzoic acid and 2-(p chlorophenoxy)-2-methylpropionic acid.BR acts coactively with active auxins (Arteca et al., 1983) and calcium, whereas it has an additive effect when used in combination with cytokinins in the stimulation of ethylene production (Arteca, 1995). Although auxin and BRs have been reported to enhance ethylene production alone or when applied in combination (Arteca et al., 1983) most of the work has been conducted in mung bean.

\section{Root growth and development}

The low concentrations of BRs such as 24epicastasterone and 24-epibrassinolide promote root elongation in Arabidopsis wild type plant up to 50\% and in BR- deficient mutant such as dwf1-6 (cbb1) and cbb3 (which is allelic to cpd) up to $150 \%$. Simultaneous administration of 24- epibrassinolide and 2,4dichorophenoxy acetic acid results in largely additive effects .Exogenous gibberellins do not promote root elongation in the BR- deficient mutant. Root growth stimulating effects of BRs appear to be largely independent of auxin and gibberalin action. BRs acts coactively with auxin to promote lateral root formation.BR with its perception is required for the transgenic expression of the $\beta$-glucouronidase gene fused to a synthetic auxin -inducible promoter in root tips; while exogenous BR promote DR5::GUS expression in the root tips and the stele region proximal to the root tips. Following their first identification in corn (Zea mays L.) roots (Kim et al., 2000). BR has been detected in the root tissues of Arabidopsis, pea and tomato (Bancos et al., 2002; Shimada et al., 2001).

\section{Plant stress to various pathogens}

Brassinosteroids are found in a diverse range of organism from lower to higher plants. Brassinosteroids metabolism has long been known to be altered in plants responding to abiotic stress and to undergo profound changes in plants interacting with bacterial, fungal and viral pathogens (Shimada et al., 2001). Brassinosteroid were found to be involved in increasing resistance to chilling in brome grass74 and rice75. In rice, 24epibrassinolide increased the resistance against chilling stress $\left(1-5^{\circ} \mathrm{C}\right)$ and the tolerance was associated with increased ATP, proline levels and SOD activity (Rao et al., 2002). In addition to their role in plant development, BRs protect plants from a variety of environmental stresses, including high and low temperatures, drought, salinity and pathogen attack. New discoveries of the physiological properties of Brassinosteroids allow us to consider them as highly promising, environment- friendly natural substances 
suitable for wide application in plant protection and yield promotion in agriculture. Most of the studies demonstrating anti-stress effects of BRs have employed treatment of plants with exogenous BRs. Although exogenous BR treatment is yet neither a conventional greenhouse nor field agricultural method, it is highly instrumental in the laboratory for studying molecular changes induced by the BR-induced molecular changes that are related to stress tolerance include higher level of expression of stress-responsive genes, maintenance of protein synthesis induction of other hormone responses, increase in the activities of antioxidant enzymes, greater accumulation of osmoprotectants and higher photosynthetic efficiency. Results of a global gene expression study of untreated and BR-treated Arabidopsis under stress and no stress conditions also indicate that BR affects a myriad of cellular processes either directly or through crosstalk with other plant hormone pathways, resulting in better performance of plants under stress conditions. Clearly then, altering endogenous BR activity through transgenic technology offers great promise of generating crops with increased stress tolerance.

\section{Possible mode of action of brassinosteroids}

The mechanism of action of BRs has been an attractive target for researchers since these elucidations of the structure of BL, but for many reasons only recently some significant progress in this direction has been achieved. Although this problem is still rather far from its final solution, however some important data have brought us closer to the understanding of the mode of regulatory action of BRs.

Considering higher variability of the physiological effects of BRs, it is believed that more than one molecular mechanisms of their action exists. Two main aspects of the primary mechanism have to be considered first: an effect of BR on the biosynthesis of enzymes via an effect on genome expression and secondly the effect of BR on membranes. The former is responsible for slow reactions of plants to the exogenous hormone, and the latter for the quick reactions. It is very well documented that steroids function as a signaling molecule both in animals and plants. While animal steroidal hormones are perceived by nuclear receptors family of transcription factors, BRs in plants are perceived by a cell surface receptor kinase, BRI1.

Brassinolide the most active brassinosteroid, in bioassays binds to the extracellular domain of BRI1 receptor. BRI1 is a plasma membrane localized leucinerich repeat (LRR)-receptor serine/threonine (S/T) kinase. The LRR-receptor kinases constitute the largest receptor class predicted in the Arabidopsis genome, with over 230 family members. This family has a conserved domain structure, composed of N-terminal extracellular domain with multiple tandem (adjacent) LRR motifs, a single trans-membrane domain, and a cytoplasmic kinase domain having specificity towards serine and threonine residues. The number of LRRs is 25 in case of BR1. BRI1 also has a unique feature that is required for BR binding, a stretch of amino acids called as island domain that interrupts the LRRs between LRRs 21 and 22 (Kinoshita, 2005). This domain plus the flanking LRR22 compose the minimum binding site for BRs. BL binding to BRI1 triggers the interaction between BRI1 and BAK1.

BRI1 is phosphorylated at multiple sites along with its intracellular domain, some of which have been shown to regulate receptor activity. The $\mathrm{BL}$ signal is then transmitted to the cytoplasm by an unknown mechanism where it inhibits BIN2, which is a negative regulator of BR biosynthetic pathway. BIN2, a protein kinase interacts with and phosphorylates two nearly identical transcription factors, BES1 and BZR1, negatively regulating their activities.

\section{Future prospects}

New discoveries of the physiological properties of Brassinosteroids allow us to consider them as highly promising, environment-friendly natural substances suitable for wide application in plant protection and yield promotion in agriculture. These data indicate that exogenous BS can act efficiently in plants as immunomodulators when applied at the appropriate dose and at the correct stage of plant development. Recent research on the chemistry, physiology, and molecular biology of BRs provides a convincing body of evidence that these plant steroids are essential regulators of plant growth and development. The pace of BR research is accelerating rapidly, and with the proliferation of cloned genes and advances in microchemical techniques, the range of experimental approaches to understanding BR action continues to expand.

\section{Conflict of interest statement}

Authors declare that they have no conflict of interest. 


\section{References}

Albrecht, C., Boutrot, F., Segonzac, C., Schwessinger, B., Gimenez-Ibanez, S., Chinchilla, D., Zipfel, C., 2012. Brassinosteroids inhibit pathogen-associated molecular pattern-triggered immune signaling independent of the receptor kinase BAK1. Proc. Nat. Acad. Sci. 109(1), 303-308.

Arteca, R.N., 1995. Brassinosteroids. In: Plant Hormones: Physiology, Biochemistry and Molecular Biology (Ed.: Davies, P. J.). Kluwer Academic Publishers, New York. pp.206-213.

Arteca, R.N., Tsai, D.S., Schlagnhaufer, C., Mandava, NB., 1983. The effects of brassinosteroid on auxininduced ethylene production by etiolated mung bean segments. Physiol. Plant. 59, 539-544.

Bai, M. Y., Shang, J. X., Oh, E., Fan, M., Bai, Y., Zentella, R., Sun, T. P., Wang, Z. Y., 2012. Brassinosteroid, gibberellin and phytochrome impinge on a common transcription module in Arabidopsis. Nat. Cell Biol. 14, 810-817.

Bailly, C., El-Maarouf-Bouteau, H., Corbineau, F., 2008. From intracellular signaling networks to cell death: The dual role of reactive oxygen species in seed physiology. C. R. Biol. 331, 806-814.

Bajguz, A., Tretyn, A., 2003. The chemical characteristic and distribution of brassinosteroids in plants. Phytochem. 62(7), 1027-1046.

Bajguz, A., 2000. Effects of brassinosteroids on nucleic acids and protein in cultured cells in Chlorella vulgaris. Plant Physiol. 38, 209-215.

Bancos, S., Nomura, T., Sato, T., Molnar, G., Bishop, G.J., Koncz, C., Yokota, T., Nagy, F., Szekeres, M., 2002. Regulation of transcript levels of the Arabidopsis cytochrome p450 genes involved in brassinosteroid biosynthesis. Plant Physiol. 130, 504-513.

Caño-Delgado, A., Yin, Y., Yu, C., Vafeados, D., MoraGarcía, S., Cheng, J.C., Chory, J., 2004. BRL1 and BRL3 are novel brassinosteroid receptors that function in vascular differentiation in Arabidopsis. Development (Cambridge, England). 131(21), 53415351.

Clay, N.K., Nelson, T., 2002. VH1, a provascular cellspecific receptor kinase that influences leaf cell patterns in Arabidopsis. Plant Cell. 14, 2707-2722.

Clouse, S.D., Sasse, J.M., 1998. Brassinosteroids: Essential regulators of plant growth and development. Annu. Rev. Plant Physiol. Plant Mol. Biol. 49(1), 427-451.

Fukaki, H., Wysocka-Diller, J., Kato, T., Fujisawa, H.,
Benfey, P.N., Tasaka, M., 1998. Genetic evidence that the endodermis is essential for shoot gravitropism in Arabidopsis thaliana. Plant J. 14(4), 425-430.

Fukuda, H., 2004. Signals that control plant vascular cell differentiation. Nature Rev. Mol. Cell Biol. 5, 379391.

Gudesblat, G.E., Russinova, E., 2011. Plants grow on brassinosteroids. Curr. Opin. Plant Biol.14, 530-537.

Hayat, S., Ahmad, A., 2011. Brassinosteroids: A Class of Plant Hormone. Springer Netherlands. DOI: 10.1007/978-94-007-0189-2

Hayat, S., Hasan, S.A., Hayat, Q., Ahmad, A., 2010. Brassinosteroids protect Lycopersicon esculentum from cadmium toxicity applied as shotgun approach. Protoplasma. 239, 3-14.

He, J.X., Gendron, J.M., Yang, Y., Li, J., Wang, Z.Y., 2002. The GSK3-like kinase BIN2 phosphorylates and destabilizes BZR1, a positive regulator of the brassinosteroid signaling pathway in Arabidopsis. Proc. Natl. Acad. Sci. (USA). 99, 10185-10190.

Hewitt, F.R., Hough, T., O’Neill, P., Sasse, J.M., Williams, E.G., Rowan, K.S., 1985. Effect of brassinolide and other growth regulators on the germination and growth of pollen tubes of Prunus avium using a multiple hanging-drop assay. Aust. J. Plant Physiol. 12, 201-211.

Jones-Held, S., Vandoren, M., Lockwood, T., 1996. Brassinolide application to Lepidium sativum seeds and the effects on seedling growth. J. Plant Growth Regul. 15(2), 63-67.

Kaur, S., Bhardwaj, R., 2004. Blockage of Znaccumulation in seedlings of Brassica campestris $\mathrm{L}$. by 24-epibrassinolide. Keystone Symposium on Plant Responses to Abiotic Stresses, Abstract No. 216: 64, Feb. 19-24.

Kim, S.K., Chang, S.C., Lee, E.J., Chung, W.S., Kim, Y.S., Hwang, S, Lee, J.S., 2000. Involvement of brassinosteroids in the gravitropic response of primary root of maize. Plant Physiol. 123, 9971004.

Kim, T.W., Guan, S., Burlingame, A.L., Wang, Z.Y., 2011. The CDG1 kinase mediates brassinosteroid signal transduction from BRI1 receptor kinase to BSU1 phosphatase and GSK3-like kinase BIN2. Mol. Cell. 43, 561-571.

Kinoshita, T., 2005. Binding of brassinosteroids to the extracellular domain of plant receptor kinase BRI1. Nature. 433, 167-171.

Li, J., Li, J., Chory, J., Chory, J., 1999. Brassinosteroid actions in plants. J. Expt. Bot. 50(332), 275-282. 
Mitchell, J.W., Mandava, N.B., Worley, J.E., Plimmer, J.R., Smith, M.V., 1970. Brassins: A family of plant hormones from rape pollen. Nature. 225, 1065-1066.

Mizukami, Y., 2001. A matter of size: Developmental control of organ size in plants. Curr. Opin. Plant Biol. 4(6), 533-539.

Nakaya, M., Tsukaya, H., Murakami, N., Kato, M., 2002. Brassinosteroids control the proliferation of leaf cells of Arabidopsis thaliana. Plant Cell Physiol. 43, 239-244.

Nemhauser, J.L., Mockler, T.C., Chory, J., 2004. Interdependency of brassinosteroid and auxin signaling in Arabidopsis. PLoS Biol. 2(9), E258.

Nomura, T., Ueno, M., Yamada, Y., Takatsuto, S., Takeuchi, Y., Yokota, T., 2007. Roles of brassinosteroids and related mRNAs in pea seed growth and germination. Plant Physiol. 143(4), 1680-1688.

Peng, P., Yan, Z., Zhu, Y., Li, J., 2008. Regulation of the Arabidopsis GSK3-like kinase BRASSINOSTEROIDINSENSITIVE 2 through proteasome-mediated protein degradation. Mol. Plant. 1(2), 338-346.

Rao, S.S.R., Vardhini, B.V., Sujatha, E., Anuradha, S., 2002. Brassinosteroids - New class of phytohormones. Curr. Sci. 82, 1239-1245.

Sasse, J.M., 2003. Physiological actions of brassinosteroids: An update. J. Plant Growth Regul. 22(4), 276-288.

Sharma, P., Bhardwaj, R., 2007. Effect of 24epibrassinolide on seed germination, seedling growth and heavy metal uptake in Brassica juncea L. Gen. Appl. Plant Physiol. 33(12), 59-73.

Shimada, Y., Fujioka, S., Miyauchi, N., Kushiro, M., Takatsuto, S., Nomura, T., Yokota, T., Kamiya, Y., Bishop, G.J., Yoshida, S., 2001. Bassinosteroid-6oxidases from Arabidopsis and tomato catalyze multiple C-6 oxidations in brassinosteroid biosynthesis. Plant Physiol. 126, 770-779.

Steber, C.M., McCourt, P., 2001, A role for brassinosteroids in germination in Arabidopsis.
Plant Physiol. 125, 763-769.

Sun, Y., Fan, X.Y.O., Cao, D.M., Tang, W, He, K., Zhu, J.Y., He, J.X., Bai, M.Y., Zhu, S., Oh, E., 2010. Integration of brassinosteroid signal transduction with the transcription network for plant growth regulation in Arabidopsis. Dev. Cell. 19, 765-777.

Suzuki, H., Fujioka, S., Takatsuto, S., Yokota, T., Murofushi, N., Sakurai, A., 1995. Biosynthesis of brassinosteroids in seedlings of Catharanthus roseus, Nicotiana tabacum, and Oryza sativa. Biosci. Biotech. Bioch. 59, 168-172.

Tang, W., Yuan, M., Wang, R., Yang, Y., Wang, C., Oses-Prieto, J. A., Kim, T.W., Zhou, H. W., Deng, Z., Gampala, S.S., 2011. PP2A activates brassinosteroid-responsive gene expression and plant growth by dephosphorylating BZR1. Nat. Cell Biol. 13, 124-131.

Tang, W., Kim, T.W., Oses-Prieto, J.A., Sun. Y., Deng, Z., Zhu, S., Wang, R., Burlingame, A.L., Wang, Z.Y., 2008. BSKs mediate signal transduction from the receptor kinase BRI1 in Arabidopsis. Science. 321, 557-560.

Volynets, A.P., Pschenichanye, L.A., Khripach, V.A., 1997. The nature of protective action of 24epibrassinolide on barley plants. Plant Growth Regul. Soc. Am. 24, 133-137.

Yamamoto, R., Demura, T., Fukuda, H., 1997. Brassinosteroids induce entry into the final stage of tracheary element differentiation in cultured Zinnia cells. Plant Cell Physiol. 38(8), 980-983.

Yokota, T., Takahashi, N., 1986. Chemistry, physiology and agricultural application of brassinolide and related steroids. In: Plant Growth Substances (Ed.: Bopp, M.). Springer-Verlag, Berlin/Heidelberg. pp.129-135.

Zhao, B., Li, J., 2012. Regulation of brassinosteroid biosynthesis and inactivation. J. Integr. Plant Biol. 54(10), 746-759.

Zhu, J.Y., Sae-Seaw, J., Wang, Z.Y., 2013. Brassinosteroid signalling. Development (Cambridge, England). 140, 1615-1620.

\section{How to cite this article:}

Khatoon, H., Ananya, S., Ahmad, F., Aisha, K., 2017. Brassinosteroids - An essential steroidal regulator: Its structure, synthesis and signaling in plant growth and development- A review. Int. J. Curr. Res. Biosci. Plant Biol. 4(7), 88-96. doi: https://doi.org/10.20546/ijcrbp.2017.407.011 